PHYSICAL REVIEW D 79, 064016 (2009)

\title{
Geodesic stability, Lyapunov exponents, and quasinormal modes
}

\author{
Vitor Cardoso* \\ Centro Multidisciplinar de Astrofísica-CENTRA, Departamento de Física, Instituto Superior Técnico, \\ Avenida Rovisco Pais 1, 1049-001 Lisboa, Portugal \\ Department of Physics and Astronomy, The University of Mississippi, University, Mississippi 38677-1848, USA \\ Alex S. Miranda ${ }^{\dagger}$ \\ Instituto de Física, Universidade Federal do Rio de Janeiro, Caixa Postal 68528, RJ, 21941-972, Brazil \\ Emanuele Berti \\ Jet Propulsion Laboratory, California Institute of Technology, Pasadena, California 91109, USA \\ Department of Physics and Astronomy, The University of Mississippi, University, Mississippi 38677-1848, USA \\ Helvi Witek ${ }^{\S}$ \\ Centro Multidisciplinar de Astrofísica-CENTRA, Departamento de Física, Instituto Superior Técnico, \\ Avenida Rovisco Pais 1, 1049-001 Lisboa, Portugal \\ Institute of Theoretical Physics, Friedrich-Schiller University Jena, Max-Wien-Platz 1, 07743 Jena, Germany \\ Vilson T. Zanchin" \\ Centro de Ciências Naturais e Humanas, Universidade Federal do ABC, Rua Santa Adélia 166, 09210-170, Santo André, SP, Brazil
}

(Received 12 December 2008; published 18 March 2009)

\begin{abstract}
Geodesic motion determines important features of spacetimes. Null unstable geodesics are closely related to the appearance of compact objects to external observers and have been associated with the characteristic modes of black holes. By computing the Lyapunov exponent, which is the inverse of the instability time scale associated with this geodesic motion, we show that, in the eikonal limit, quasinormal modes of black holes in any dimensions are determined by the parameters of the circular null geodesics. This result is independent of the field equations and only assumes a stationary, spherically symmetric and asymptotically flat line element, but it does not seem to be easily extendable to anti-de Sitter spacetimes. We further show that (i) in spacetime dimensions greater than four, equatorial circular timelike geodesics in a Myers-Perry black-hole background are unstable, and (ii) the instability time scale of equatorial null geodesics in Myers-Perry spacetimes has a local minimum for spacetimes of dimension $d \geq 6$.
\end{abstract}

DOI: 10.1103/PhysRevD.79.064016

PACS numbers: 04.70.Bw, 04.50.Gh, 05.45.- a

\section{INTRODUCTION}

Geodesics in black-hole spacetimes have been extensively studied, both in four- and higher-dimensional spacetimes, with and without a cosmological constant. Geodesics may display a rich structure and they convey important information on the background geometry. Among the different kinds of geodesic motion, circular geodesics are especially interesting. For instance, the binding energy of the last stable circular timelike geodesic in the Kerr geometry is related to the gravitational binding energy that can be radiated to infinity, and it can be used to estimate the spin of astrophysical black holes through observations of accretion disks [1-3].

It was shown many years ago that null geodesics also play an important role. The optical appearance of a star

\footnotetext{
*vcardoso@fisica.ist.utl.pt

†astmiranda@if.ufrj.br

*berti@wugrav.wustl.edu

\$helvi.witek@ist.utl.pt

"zanchin@ufabc.edu.br
}

undergoing gravitational collapse depends crucially on the circular unstable null geodesic, which also explains an exponential fade-out of the collapsing star's luminosity $[4,5]$. Null geodesics are also very useful to explain the characteristic modes of a black hole - the so-called quasinormal modes (QNMs) [6,7]. These "free" modes of vibration can be interpreted in terms of null particles trapped at the unstable circular orbit and slowly leaking out [8-12]. The real part of the complex QNM frequencies is determined by the angular velocity at the unstable null geodesic; the imaginary part is related to the instability time scale of the orbit, a quantity which is seldom considered in geodesic studies, with some noteworthy exceptions (see, e.g., [13-19]). Furthermore, there is some evidence [15] that unstable circular orbits could yield information on phenomena occurring at the threshold of black-hole formation in the high-energy scattering of black holes, a process of interest in fundamental physics for a variety of reasons [20,21].

In this work we clarify some aspects of the relation among unstable null geodesics, Lyapunov exponents and 
quasinormal modes. In Sec. II we derive a simple formula for the principal Lyapunov exponent $\lambda$ in terms of the second derivative of the effective potential for radial motion $V_{r}$ :

$$
\lambda=\sqrt{\frac{V_{r}^{\prime \prime}}{2 \dot{t}^{2}}}
$$

where $t$ is coordinate time. Throughout this work, a dot denotes a derivative with respect to proper time and a prime stands for a derivative with respect to areal radius $r$. The result above is valid for a wide class of spacetimes and geodesics, including stationary spherically symmetric spacetimes and equatorial orbits in the geometry of higher-dimensional, rotating (Myers-Perry) black-hole solutions.

In Sec. III we show that the relation between QNMs and unstable circular null geodesics is quite general, being valid in the eikonal limit for any static spherically symmetric, asymptotically flat spacetime. More specifically, we show that the angular velocity $\Omega_{c}$ at the unstable null geodesic and the Lyapunov exponent, determining the instability time scale of the orbit (see, for instance, $[13,14])$ agree with analytic WKB approximations for QNMs [22-24]:

$$
\omega_{\mathrm{QNM}}=\Omega_{c} l-i(n+1 / 2)|\lambda|,
$$

where $n$ is the overtone number and $l$ is the angular momentum of the perturbation. The WKB results are formally valid only in the eikonal regime $(l \gg 1)$, but they seem to yield surprisingly accurate predictions even for low values of $l[12,25]$. A simple derivation of the Lyapunov exponent for spherically symmetric, asymptotically flat spacetimes, patterned after the original QNM calculation by Ferrari and Mashhoon [10,11], is provided in Appendix A. For the important case of a $d$-dimensional Schwarzschild-Tangherlini [26] black-hole solution we find that the critical exponent defined by Pretorius and Khurana [15] can be determined analytically to be

$$
\gamma \equiv \frac{\Omega_{c}}{2 \pi \lambda}=\frac{1}{2 \pi \sqrt{d-3}} .
$$

By exploring the connection between QNMs and null geodesics, we also find a simple analytical result for the quasinormal frequencies of near-extremal Schwarzschildde Sitter black holes in $d=4$ :

$$
\omega_{\mathrm{QNM}}=\kappa_{+}[l-i(n+1 / 2)],
$$

where $\kappa_{+}$denotes the surface gravity. In the eikonal limit, the above result agrees with that found in [27].

In Sec. IV we analyze the higher-dimensional rotating black-hole solutions found by Myers and Perry [28]. In $d=5$ we can compute $\lambda$ analytically. The Lyapunov exponent goes to zero as one approaches extremality in $d=$ 4, 5 spacetime dimensions. However, no such behavior is observed for $d>5$ : the Lyapunov exponent (normalized by the orbital frequency) has a local minimum, which may be related to a possible instability of the system first suggested by Emparan and Myers [29]. In Appendix B we study in some detail timelike circular geodesics in the Myers-Perry spacetime and show that equatorial circular orbits are always unstable for $d>4$. Finally, in Appendix $\mathrm{C}$ we discuss issues in generalizing our results to non-asymptotically flat spacetimes.

\section{LYAPUNOV EXPONENTS AND GEODESIC STABILITY}

Lyapunov exponents are a measure of the average rate at which nearby trajectories converge or diverge in the phase space. A positive Lyapunov exponent indicates a divergence between nearby trajectories, i.e., a high sensitivity to initial conditions. A geodesic stability analysis in terms of Lyapunov exponents begins with the equations of motion schematically written as

$$
\frac{d X_{i}}{d t}=H_{i}\left(X_{j}\right)
$$

and linearized about a certain orbit:

$$
\frac{d \delta X_{i}(t)}{d t}=K_{i j}(t) \delta X_{j}(t)
$$

Here

$$
K_{i j}(t)=\left.\frac{\partial H_{i}}{\partial X_{j}}\right|_{X_{i}(t)}
$$

is the linear stability matrix [14]. The solution to the linearized equations can be written as

$$
\delta X_{i}(t)=L_{i j}(t) \delta X_{j}(0)
$$

in terms of the evolution matrix $L_{i j}(t)$, which must obey

$$
\dot{L}_{i j}(t)=K_{i m} L_{m j}(t)
$$

and $L_{i j}(0)=\delta_{i j}$. A determination of the eigenvalues of $L_{i j}$ leads to the principal Lyapunov exponent $\lambda$, which is the quantity we focus on. Specifically

$$
\lambda=\lim _{t \rightarrow \infty} \frac{1}{t} \log \left(\frac{L_{j j}(t)}{L_{j j}(0)}\right) .
$$

We now restrict attention to a class of problems for which one has a two-dimensional phase space of the form $X_{i}(t)=\left(p_{r}, r\right)$. This includes circular orbits in stationary spherically symmetric spacetimes and equatorial circular orbits in stationary spacetimes, such as the MyersPerry metric considered in Sec. IV. Linearizing the equations of motion with $X_{i}(t)=\left(p_{r}, r\right)$ about orbits of constant $r$ we get

$$
K_{i j}=\left(\begin{array}{cc}
0 & K_{1} \\
K_{2} & 0
\end{array}\right),
$$

where 


$$
\begin{gathered}
K_{1}=\frac{d}{d r}\left(\dot{t}^{-1} \frac{\delta \mathcal{L}}{\delta r}\right), \\
K_{2}=-\left(\dot{t}_{r r}\right)^{-1},
\end{gathered}
$$

and $\mathcal{L}$ is the Lagrangian for geodesic motion (see below for explicit examples). Therefore, for circular orbits, the principal Lyapunov exponents can be expressed as

$$
\lambda= \pm \sqrt{K_{1} K_{2}}
$$

From now on we will drop the \pm sign, and simply refer to the "Lyapunov exponent." From the equations of motion it follows that

$$
\frac{d}{d \tau} \frac{\delta \mathcal{L}}{\delta \dot{r}}=\frac{\delta \mathcal{L}}{\delta r}
$$

and

$$
\frac{d}{d \tau} \frac{\delta \mathcal{L}}{\delta \dot{r}}=\frac{d}{d \tau}\left(-g_{r r} \dot{r}\right)=-\dot{r} \frac{d}{d r}\left(g_{r r} \dot{r}\right) .
$$

Using the definition of $V_{r}$

$$
\dot{r}^{2}=V_{r} \text {, }
$$

we can rewrite this as

$$
\frac{\delta \mathcal{L}}{\delta r}=-\frac{1}{2 g_{r r}} \frac{d}{d r}\left(g_{r r}^{2} V_{r}\right) .
$$

For circular geodesics $V_{r}=V_{r}^{\prime}=0$ [30], and Eq. (14) reduces to

$$
\lambda=\sqrt{\frac{V_{r}^{\prime \prime}}{2 \dot{t}^{2}}}
$$

Following Pretorius and Khurana [15], we can define a critical exponent

$$
\gamma \equiv \frac{\Omega_{c}}{2 \pi \lambda}=\frac{T_{\lambda}}{T_{\Omega}}
$$

where we introduced a typical orbital time scale $T_{\Omega} \equiv$ $2 \pi / \Omega_{c}$ and an instability time scale $T_{\lambda} \equiv 1 / \lambda$ (note that in Ref. [14] the authors use a different definition of the orbital time scale, $T_{\Omega} \equiv 2 \pi / \dot{\varphi}$, with $\varphi$ an angular coordinate). Then we get

$$
\gamma=\frac{1}{2 \pi} \sqrt{\frac{\dot{\varphi}^{2}}{2 V_{r}^{\prime \prime}}}
$$

For circular null geodesics in many spacetimes of interest $V_{r}^{\prime \prime}>0$, which implies instability. A quantitative characterization of this instability can be achieved by computing the time scale associated with it. In most of this paper we will use the method outlined above (see also [13,31,32]), but there are alternative approaches [10,11,14,33]. In Appendix A, for example, we recover the results of the next section following a stability analysis due to Ferrari and Mashhoon [10,11].
The discussion above is still rather general, assuming only that the variables in the equations of motion form a two-dimensional plane in phase space. We now specialize to spherically symmetric spacetimes.

\section{SPHERICALLY SYMMETRIC SPACETIMES}

We will consider a stationary spherically symmetric background

$$
d s^{2}=f(r) d t^{2}-\frac{1}{g(r)} d r^{2}-r^{2} d \Omega_{d-2}^{2},
$$

where $f(r)$ and $g(r)$ are functions to be determined by solving the field equations, $d \Omega_{d-2}^{2}$ is the metric of the $(d-2)$-sphere and $A_{d-2} \equiv 2 \pi^{(d-1) / 2} / \Gamma[(d-1) / 2]$ is the area of the unit $(d-2)$-sphere. Since we do not specify the field equations, our results are valid for any field equations admitting spherically symmetric, asymptotically flat solutions. The last property will be required to enforce the correct boundary conditions in the WKB calculations of Sec. III C.

\section{A. Circular orbits}

To compute the geodesics in the spacetime (22) we follow Chandrasekhar [34]. Let us restrict attention to equatorial orbits, for which the Lagrangian is

$$
2 \mathcal{L}=f(r) \dot{t}^{2}-\frac{1}{g(r)} \dot{r}^{2}-r^{2} \dot{\varphi}^{2},
$$

where $\varphi$ is an angular coordinate. The generalized momenta derived from this Lagrangian are

$$
\begin{gathered}
p_{t}=f(r) \dot{t} \equiv E=\text { const } \\
p_{\varphi}=-r^{2} \dot{\varphi} \equiv-L=\text { const, } \\
p_{r}=-\frac{1}{g(r)} \dot{r} .
\end{gathered}
$$

The Lagrangian is independent of both $t$ and $\varphi$, so it follows immediately that $p_{t}$ and $p_{\varphi}$ are two integrals of motion. Solving (24) and (25) for $\dot{t}$ and $\dot{\varphi}$ we get

$$
\dot{\varphi}=\frac{L}{r^{2}}, \quad \dot{t}=\frac{E}{f(r)} .
$$

The Hamiltonian is given by

$$
\begin{aligned}
2 \mathcal{H} & =2\left(p_{t} \dot{t}+p_{\varphi} \dot{\varphi}+p_{r} \dot{r}-\mathcal{L}\right) \\
& =f(r) \dot{t}^{2}-\frac{1}{g(r)} \dot{r}^{2}-r^{2} \dot{\varphi}^{2} \\
& =E \dot{t}-L \dot{\varphi}-\frac{1}{g(r)} \dot{r}^{2}=\delta_{1}=\text { const. }
\end{aligned}
$$

Here $\delta_{1}=1,0$ for timelike and null geodesics, respectively. Inserting Eq. (27) in (28) and using the definition (17) we get 


$$
V_{r}=g(r)\left[\frac{E^{2}}{f(r)}-\frac{L^{2}}{r^{2}}-\delta_{1}\right] .
$$

\section{Timelike geodesics}

The requirement $V_{r}=V_{r}^{\prime}=0$ for circular orbits yields

$$
E^{2}=\frac{2 f^{2}}{2 f-r f^{\prime}}, \quad L^{2}=\frac{r^{3} f^{\prime}}{2 f-r f^{\prime}},
$$

where here and below all quantities are evaluated at the radius of a circular timelike orbit. Since the energy must be real, we require

$$
2 f-r f^{\prime}>0 .
$$

The second derivative of the potential is

$$
V_{r}^{\prime \prime}=2 \frac{g}{f} \frac{-3 f f^{\prime} / r+2\left(f^{\prime}\right)^{2}-f f^{\prime \prime}}{2 f-r f^{\prime}},
$$

and the orbital angular velocity is given by

$$
\Omega=\frac{\dot{\varphi}}{\dot{t}}=\left(\frac{f^{\prime}}{2 r}\right)^{1 / 2} .
$$

\section{Null geodesics}

Circular null geodesics satisfy the conditions

$$
\begin{aligned}
& \frac{E}{L}= \pm \sqrt{\frac{f_{c}}{r_{c}^{2}}}, \\
& 2 f_{c}=r_{c} f_{c}^{\prime} .
\end{aligned}
$$

Here and below a subscript $c$ means that the quantity in question is evaluated at the radius $r=r_{c}$ of a circular null geodesic. An inspection of (35) shows that circular null geodesics can be seen as the innermost circular timelike geodesics. In this case

$$
V_{r}^{\prime \prime}\left(r_{c}\right)=\frac{L^{2} g_{c}}{r_{c}^{4} f_{c}}\left[2 f_{c}-r_{c}^{2} f_{c}^{\prime \prime}\right],
$$

and the coordinate angular velocity is

$$
\Omega_{c}=\frac{\dot{\varphi}}{\dot{t}}=\left(\frac{f_{c}^{\prime}}{2 r_{c}}\right)^{1 / 2}=\frac{f_{c}^{1 / 2}}{r_{c}} .
$$

\section{B. Lyapunov exponents}

\section{Timelike geodesics}

Using Eqs. (19), (27), and (32) to evaluate the Lyapunov exponent at the circular timelike geodesics, we get

$$
\begin{aligned}
\lambda & =\frac{1}{\sqrt{2}} \sqrt{-\frac{g}{f}\left[\frac{3 f f^{\prime}}{r}-2\left(f^{\prime}\right)^{2}+f f^{\prime \prime}\right]} \\
& =\frac{1}{2} \sqrt{\left(2 f-r f^{\prime}\right) V_{r}^{\prime \prime}(r) .}
\end{aligned}
$$

Bearing in mind that $2 f-r f^{\prime}>0$ and that unstable orbits are defined by $V_{r}^{\prime \prime}>0$, we can see that $\lambda$ will be real whenever the orbit is unstable, as expected. In $d=4$ this formula reduces to well-known results [14]. We also get

$$
\frac{1}{\gamma^{2}}=\left(\frac{2 \pi \lambda}{\Omega}\right)^{2}=(2 \pi)^{2}\left[-3 g+2 r \frac{g}{f} f^{\prime}-r \frac{g f^{\prime \prime}}{f^{\prime}}\right] .
$$

\section{Null geodesics}

Using Eqs. (19), (27), (34), and (36), for circular null geodesics we find

$$
\lambda=\frac{1}{\sqrt{2}} \sqrt{\frac{r_{c}^{2} f_{c}}{L^{2}} V_{r}^{\prime \prime}\left(r_{c}\right)}=\frac{1}{\sqrt{2}} \sqrt{-\frac{r_{c}^{2}}{f_{c}}\left(\frac{d^{2}}{d r_{*}^{2}} \frac{f}{r^{2}}\right)_{r=r_{c}} .} .
$$

In the last equality we made use of (35) and we defined the "tortoise" coordinate

$$
\frac{d r}{d r_{*}}=\sqrt{g(r) f(r)} .
$$

\section{Unstable null geodesics and quasinormal modes: Comparison with WKB results}

WKB methods [22-25] provide an accurate approximation of QNM frequencies in the eikonal limit for spacetimes where the wave equation can be cast in the form

$$
\frac{d^{2}}{d r_{*}^{2}} \Psi+Q_{0} \Psi=0
$$

where $r_{*}$ is a convenient tortoise coordinate, ranging from $-\infty$ to $+\infty$. In particular, one gets the QNM condition

$$
\frac{Q_{0}\left(r_{0}\right)}{\sqrt{2 Q_{0}^{(2)}\left(r_{0}\right)}}=i(n+1 / 2),
$$

where $Q_{0}^{(2)} \equiv d^{2} Q_{0} / d r_{*}^{2}$ and Eq. (43) is evaluated at the extremum of $Q_{0}$, i.e., the point $r_{0}$ at which $d Q_{0} / d r_{*}=0$. We note that this result is strictly valid only for asymptotically flat spacetimes, or for spacetimes admitting wavelike solutions at spatial infinity. It is not valid for anti-de Sitter backgrounds. In a spacetime of the form (22), we find that the Klein-Gordon equation can be written as in Eq. (42) with the tortoise coordinate (41). In the eikonal limit $(l \rightarrow$ $\infty)$ we get

$$
Q_{0} \simeq \omega^{2}-f \frac{l^{2}}{r^{2}} .
$$

It is known that scalar, electromagnetic and gravitational perturbations of static black holes in higher dimensions have the same behavior in the eikonal limit [35-37]. In other words, there is a well-defined geometric-optics (eikonal) limit where the potential for a wide class of massless perturbations is "universal." For $Q_{0}$ above we find that the extremum of $Q_{0}$ satisfies $2 f\left(r_{0}\right)=r_{0} f^{\prime}\left(r_{0}\right)$, i.e., $r_{0}$ 
coincides with the location of the null circular geodesic $r_{0}=r_{c}$, as given by Eq. (35). Furthermore, the WKB formula (43) allows one to conclude that, in the large- $l$ limit,

$$
\omega_{\mathrm{QNM}}=l \sqrt{\frac{f_{c}}{r_{c}^{2}}}-i \frac{(n+1 / 2)}{\sqrt{2}} \sqrt{-\frac{r_{c}^{2}}{f_{c}}\left(\frac{d^{2}}{d r_{*}^{2}} \frac{f(r)}{r^{2}}\right)_{r=r_{c}}} .
$$

Comparing with Eqs. (37) and (40) we find that

$$
\omega_{\mathrm{QNM}}=\Omega_{c} l-i(n+1 / 2)|\lambda| .
$$

This is one of the main results of this paper: in the eikonal approximation, the real and imaginary parts of the QNMs of any spherically symmetric, asymptotically flat spacetime are given by (multiples of) the frequency and instability time scale of the unstable circular null geodesics.

\section{Higher-dimensional Schwarzschild black hole}

Let us consider a more specific example: the higherdimensional Schwarzschild-Tangherlini metric [26]

$$
\begin{aligned}
& d s^{2}=f d t^{2}-f^{-1} d r^{2}-r^{2} d \Omega_{d-2}^{2}, \\
& f(r)=1-\left(\frac{r_{+}}{r}\right)^{d-3},
\end{aligned}
$$

which includes the well-known four-dimensional geometry as a special case. Here $d \Omega_{d-2}^{2}$ is the metric of the $(d-2)$ sphere, and the horizon radius $r_{+}$is related to the mass $M$ of the spacetime via $M=(d-2) A_{d-2} r_{+}^{d-3} /(16 \pi)$. For timelike geodesics we find that the orbits must satisfy

$$
r>r_{c}=\left(\frac{d-1}{2}\right)^{1 /(d-3)} r_{+},
$$

where $r_{c}$ is the radius of the circular null geodesic. With the requirement (31) we have $V_{r}^{\prime \prime}>0$ for all $d>4$, and therefore all circular orbits are unstable for $d>4$ [26,38]. The four-dimensional case is special: one gets $V_{r}^{\prime \prime}=$ $4 M r(6 M-r) /(2 r-6 M)$. Thus, in four spacetime dimensions there are stable circular orbits for any $r>6 M$. The circular orbits with radius $3 M<r<6 M$ are all unstable. For lightlike geodesics, one has $V_{r}^{\prime \prime}\left(r_{c}\right)=L^{2}(2 d-$ 6) $r_{c}^{-4}>0$. Therefore circular null geodesics are always unstable for $d \geq 4$. The angular velocity at $r_{c}$ is given by

$$
\Omega_{c}^{2}=\frac{d-3}{2}\left[\frac{2}{d-1}\right]^{(d-1) /(d-3)} \frac{1}{r_{+}^{2}} .
$$

The calculation of the Pretorius-Khurana [15] critical exponent yields

$$
\gamma \equiv \frac{\Omega_{c}}{2 \pi \lambda}=\frac{T_{\lambda}}{T_{\Omega}}=\frac{1}{2 \pi \sqrt{d-3}},
$$

where in the last equality we made use of Eq. (49). This result is in excellent agreement with numerical calculations by Merrick and Pretorius [39]. Small values of $\gamma$ correspond to a strong Lyapunov instability, so the insta- bility is more pronounced for large spacetime dimensions. By relating the geodesic orbital frequency and instability time scale to the QNM frequencies in the eikonal limit, we get

$$
\frac{\omega_{\mathrm{QNM}}}{\Omega_{c}}=l-i \sqrt{d-3}(n+1 / 2) .
$$

This is in complete agreement with known analytical and numerical results in $d=4$ [40] and higher dimensions [41-43], for asymptotically flat spacetimes.

\section{Near-extremal Schwarzschild-de Sitter spacetime in four dimensions}

A nontrivial example concerns a non-asymptotically flat spacetime, the near-extremal Schwarzschild-de Sitter $(\mathrm{SdS})$ spacetime in four dimensions. General SdS spacetimes have a metric of the form (22) with $f(r)=g(r)=$ $1-2 M / r-\left(r / L_{\mathrm{dS}}\right)^{2} . M$ denotes the black-hole mass and $L_{\mathrm{dS}}^{2}$ is the de Sitter curvature radius, related to the cosmological constant $\Lambda$ by $L_{\mathrm{dS}}^{2}=3 / \Lambda$. The spacetime possesses two horizons: the black-hole horizon is at $r=r_{+}$ and the cosmological horizon is at $r=r_{\mathrm{Co}}$, where $r_{\mathrm{Co}}>$ $r_{+}$. The function $f$ has zeros at $r_{+}, r_{\mathrm{Co}}$, and $r_{0}=-\left(r_{+}+\right.$ $\left.r_{\mathrm{Co}}\right)$. In terms of these quantities, $f$ can be expressed as

$$
f=\frac{1}{L_{\mathrm{dS}}^{2} r}\left(r-r_{+}\right)\left(r_{\mathrm{Co}}-r\right)\left(r-r_{0}\right) .
$$

It is useful to regard $r_{+}$and $r_{\mathrm{Co}}$ as the two fundamental parameters of the $\mathrm{SdS}$ spacetime, and to express $M$ and $L_{\mathrm{dS}}^{2}$ as functions of these variables:

$$
\begin{gathered}
L_{\mathrm{dS}}^{2}=r_{+}^{2}+r_{+} r_{\mathrm{Co}}+r_{\mathrm{Co}}^{2}, \\
2 M L_{\mathrm{dS}}^{2}=r_{+} r_{\mathrm{Co}}\left(r_{+}+r_{\mathrm{Co}}\right) .
\end{gathered}
$$

We also introduce the surface gravity $\kappa_{+}$associated with the black-hole horizon $r=r_{+}: \quad \kappa_{+} \equiv \frac{1}{2}(d f / d r)_{r=r_{+}}$. Explicitly, we have

$$
\kappa_{+}=\frac{\left(r_{\mathrm{Co}}-r_{+}\right)\left(r_{+}-r_{0}\right)}{2 L_{\mathrm{dS}}^{2} r_{+}} .
$$

Let us now specialize to the near-extremal SdS black hole, which is defined as the spacetime for which the cosmological horizon $r_{\mathrm{Co}}$ is very close (in the $r$ coordinate) to the black-hole horizon $r_{+}$, i.e.,

$$
r_{\mathrm{Co}}-r_{+} \ll r_{+} .
$$

For this spacetime one can make the following approximations:

$$
r_{0} \sim-2 r_{+}^{2} ; \quad L_{\mathrm{dS}}^{2} \sim 3 r_{+}^{2} ; \quad M \sim \frac{r_{+}}{3} ; \quad \kappa_{+} \sim \frac{r_{\mathrm{Co}}-r_{+}}{2 r_{+}^{2}} .
$$

Note that $\kappa_{+}$is infinitesimally small, since we assume Eq. (56) holds. For null geodesics, an exact solution can be 
found with

$$
r_{c}=\frac{3}{2}\left(1-\frac{r_{+}^{2}}{L_{\mathrm{dS}}^{2}}\right) r_{+} .
$$

The angular velocity at this radius is given by

$$
L_{\mathrm{dS}}^{2} \Omega_{c}^{2}=-1+\frac{4}{27}\left(1-\frac{r_{+}^{2}}{L_{\mathrm{dS}}^{2}}\right)^{-2} \frac{L_{\mathrm{dS}}^{2}}{r_{+}^{2}}
$$

and reduces to

$$
\Omega_{c}=\frac{\left(r_{\mathrm{Co}}-r_{+}\right)}{2 r_{+}^{2}}=\kappa_{+}
$$

in the near-extremal regime. As in the case of fourdimensional Schwarzschild black holes, Eq. (50), we find

$$
\lambda / \Omega_{c}=1 \text {. }
$$

This formula predicts

$$
\omega_{\mathrm{QNM}}=\kappa_{+}[l-i(n+1 / 2)] .
$$

The QNMs of this spacetime are known in closed form [27] and agree with (62) in the eikonal limit.

\section{MYERS-PERRY BLACK HOLES}

In four dimensions there is only one possible rotation axis for an axisymmetric spacetime, and there is therefore only one angular momentum parameter. In higher dimensions there are several choices of rotation axis and there is a multitude of angular momentum parameters, each referring to a particular rotation plane. Rotating black-hole solutions in higher dimensions are known as Myers-Perry black holes [28]. We focus on the simplest case for which there is only one angular momentum parameter, that we shall denote by $a$. The metric of a $d$-dimensional MyersPerry black hole with only one nonzero angular momentum parameter in Boyer-Lindquist-type coordinates is given by (here we adopt the notation commonly used in related works [43-47])

$$
\begin{aligned}
d s^{2}= & \frac{\Delta-a^{2} \sin ^{2} \vartheta}{\Sigma} d t^{2}+\frac{2 a\left(r^{2}+a^{2}-\Delta\right) \sin ^{2} \vartheta}{\Sigma} d t d \varphi \\
& -\frac{\left(r^{2}+a^{2}\right)^{2}-\Delta a^{2} \sin ^{2} \vartheta}{\Sigma} \sin ^{2} \vartheta d \varphi^{2}-\frac{\Sigma}{\Delta} d r^{2} \\
& -\Sigma d \vartheta^{2}-r^{2} \cos ^{2} \vartheta d \Omega_{d-4}^{2},
\end{aligned}
$$

where

$$
\Sigma=r^{2}+a^{2} \cos ^{2} \vartheta, \quad \Delta=r^{2}+a^{2}-\mu r^{5-d},
$$

and $d \Omega_{d-4}^{2}$ denotes the standard metric of the unit $(d-4)$ sphere [28]. This metric describes a rotating black hole in an asymptotically flat, vacuum spacetime with mass and angular momentum proportional to $\mu$ and $\mu a$, respectively. Hereafter we assume $\mu>0$ and $a>0$.

The event horizon is located at $r=r_{+}$such that $\left.\Delta\right|_{r=r_{+}}=0$. In the standard four-dimensional case, an event horizon exists only for $a<\mu / 2$. In $d=5$ an event horizon exists only when $a<\sqrt{\mu}$, and it shrinks to zero area in the extreme limit $a \rightarrow \sqrt{\mu}$. On the other hand, when $d \geq 6$, which is the part of the parameter space that we shall focus on, $\Delta=0$ has exactly one positive root for arbitrary $a>0$. This means there is no bound on $a$, or (loosely speaking) that there are no extremal rotating black holes in higher dimensions.

\section{A. Circular geodesics in the equatorial plane}

To write down the geodesic equations in the MyersPerry spacetime we follow Chandrasekhar [34]. Let us restrict attention to orbits in the equatorial plane $(\dot{\vartheta}=0$, $\vartheta=\pi / 2$ ), for which the appropriate Lagrangian is

$$
2 \mathcal{L}=g_{t t} \dot{t}^{2}+2 g_{t \varphi} \dot{t} \dot{\varphi}+g_{r r} \dot{r}^{2}+g_{\varphi \varphi} \dot{\varphi}^{2} .
$$

The generalized momenta following from this Lagrangian are

$$
\begin{gathered}
p_{t}=g_{t t} \dot{t}+g_{t \varphi} \dot{\varphi} \equiv E=\mathrm{const}, \\
p_{\varphi}=g_{t \varphi} \dot{t}+g_{\varphi \varphi} \dot{\varphi} \equiv-L=\mathrm{const}, \\
p_{r}=g_{r r} \dot{r} .
\end{gathered}
$$

The Lagrangian is independent of both $t$ and $\varphi$, so it follows immediately that $p_{t}$ and $p_{\varphi}$ are two integrals of motion. Solving (66) and (67) for $\dot{t}$ and $\dot{\varphi}$ we find

$$
\begin{gathered}
\dot{\varphi}=\frac{1}{\Delta}\left[\frac{a \mu}{r^{d-3}} E+\left(1-\frac{\mu}{r^{d-3}}\right) L\right], \\
\dot{t}=\frac{1}{\Delta}\left[\left(r^{2}+a^{2}+\frac{a^{2} \mu}{r^{d-3}}\right) E-\frac{a \mu}{r^{d-3}} L\right] .
\end{gathered}
$$

The Hamiltonian is given by

$$
\begin{aligned}
2 \mathcal{H}= & 2\left(p_{t} \dot{t}+p_{\varphi} \dot{\varphi}+p_{r} \dot{r}-\mathcal{L}\right) \\
= & \left(1-\frac{\mu}{r^{d-3}}\right) \dot{t}^{2}+\frac{2 a \mu}{r^{d-3}} \dot{t} \dot{\varphi}-\frac{r^{2}}{\Delta} \dot{r}^{2} \\
& -\left(r^{2}+a^{2}+\frac{a^{2} \mu}{r^{d-3}}\right) \dot{\varphi}^{2} \\
= & E \dot{t}-L \dot{\varphi}-\frac{r^{2}}{\Delta} \dot{r}^{2}=\delta_{1}=\text { const. }
\end{aligned}
$$

Here $\delta_{1}=1,0$ for timelike and null geodesics, respectively. Inserting Eqs. (69) and (70) in Eq. (71) we get

$$
\begin{aligned}
\dot{r}^{2} & =V_{r}, \\
r^{2} V_{r} & =\left[r^{2} E^{2}+\frac{\mu}{r^{d-3}}(a E-L)^{2}+\left(a^{2} E^{2}-L^{2}\right)-\delta_{1} \Delta\right] .
\end{aligned}
$$

In $d=4$, recalling that $\mu=2 M$, we recover the wellknown results for the Kerr geometry [34]. In five dimensions we recover the results by Frolov and Stojkovic [48], 
if we specialize their equations to only one rotation parameter.

The conditions for the existence of circular orbits, $V_{r}=$ $V_{r}^{\prime}=0$, lead to the following equations:

$$
\begin{aligned}
0= & r^{2} E^{2}+\mu r^{3-d}(a E-L)^{2}+\left(a^{2} E^{2}-L^{2}\right)-\delta_{1} \Delta, \\
0= & 4 r^{2} E^{2}-(d-5) \mu r^{3-d}(a E-L)^{2}+2\left(a^{2} E^{2}-L^{2}\right) \\
& -\delta_{1}\left(2 \Delta+r \Delta^{\prime}\right) .
\end{aligned}
$$

Eliminating the term $a^{2} E^{2}-L^{2}$ we get

$$
2 r E^{2}-(d-3) \mu r^{2-d}(a E-L)^{2}-\Delta^{\prime} \delta_{1}=0 .
$$

\section{B. Circular null geodesics}

For lightlike geodesics $\left(\delta_{1}=0\right)$ we get the explicit conditions

$$
\begin{gathered}
0=r_{c}^{2} E^{2}+\mu r_{c}^{3-d}(a E-L)^{2}+\left(a^{2} E^{2}-L^{2}\right), \\
0=2 r_{c} E^{2}-(d-3) r_{c}^{2-d} \mu(a E-L)^{2} .
\end{gathered}
$$

The above equations can be simplified by the introduction of the impact parameter $D_{c}=L / E$ :

$$
\begin{gathered}
0=r_{c}^{2}+\mu r_{c}^{3-d}\left(a-D_{c}\right)^{2}+\left(a^{2}-D_{c}^{2}\right), \\
0=2 r_{c}-(d-3) \mu r_{c}^{2-d}\left(a-D_{c}\right)^{2} .
\end{gathered}
$$

From Eq. (78) we get

$$
D_{c}=a \mp \sqrt{\frac{2 r_{c}^{d-1}}{(d-3) \mu}} .
$$

Notice that Eq. (77) is satisfied if and only if $\left|D_{c}\right|>a$. For counterrotating orbits, we have $\left|D_{c}-a\right|=-\left(D_{c}-a\right)$ and this case corresponds to the upper sign in the above equation, while for corotating orbits, $\left|D_{c}-a\right|=+\left(D_{c}-\right.$ a) and this case corresponds to the lower sign in Eq. (79).

Substituting Eq. (79) in (77), we find an equation for the radius of circular null geodesics:

$$
\frac{d-1}{d-3} r_{c}^{2} \pm 2 a \sqrt{\frac{2 r_{c}^{d-1}}{(d-3) \mu}}-\frac{2 r_{c}^{d-1}}{(d-3) \mu}=0 .
$$

In $d=4$ we recover the well-known results [30].

An important quantity for the analysis of the null geodesics is the angular frequency at the null geodesic $\Omega_{c}$ :

$$
\Omega_{c}=\frac{a \mu r_{c}^{3-d}+\left(1-\mu r_{c}^{3-d}\right) D_{c}}{\left(r_{c}^{2}+a^{2}+a^{2} \mu r_{c}^{3-d}\right)-a \mu r_{c}^{3-d} D_{c}}=\frac{1}{D_{c}},
$$

where we have used Eqs. (79) and (80). Therefore the frequency of equatorial null geodesics is the inverse of their impact parameter. This generalizes the fourdimensional result [34] to the general case of MyersPerry spacetimes. It would be interesting to investigate whether or not this is a general property of any stationary spacetime.
For corotating orbits with $a \gg \mu^{1 /(d-3)}$ and $d>5$, the following analytical approximations are valid:

$$
\begin{aligned}
r_{\mathrm{c}}^{(d-5) / 2} & \approx \frac{d-1}{2 a \sqrt{2} \sqrt{\frac{\mu}{d-3}}}, \\
D_{c}-a & \approx \sqrt{\frac{2}{\mu(d-3)}}\left(\frac{d-1}{2 a \sqrt{2}} \sqrt{\frac{\mu}{d-3}}\right)^{(d-1) /(d-5)} \\
& (\text { corotating, } a \rightarrow \infty) .
\end{aligned}
$$

In particular, when the rotation is very large the radius of the corotating orbit is at fixed relative distance from the horizon:

$$
\left(\frac{r_{c}^{\mathrm{co}}}{r_{+}}\right)^{(d-5) / 2} \rightarrow \frac{d-1}{2 \sqrt{2(d-3)}} .
$$

This may be explained by the fact that the angular velocity of the horizon $\Omega_{r_{+}}=\frac{a}{r_{+}^{2}+a^{2}}$ also has a maximum and then decreases to zero for very large $a$.

On the other hand, counterrotating orbits are well described by

$$
\begin{gathered}
r_{c}^{(d-1) / 2} \approx a \sqrt{2 \mu(d-3)}, \quad D_{c}=\frac{1}{\Omega_{c}}=-a \\
\text { (counterrotating, } a \rightarrow \infty) .
\end{gathered}
$$

We can express the radius of counterrotating orbits in terms of the horizon radius in the limit of very large rotation:

$$
\left(\frac{r_{c}^{\text {counter }}}{r_{+}}\right)^{(d-1) / 2} \rightarrow \frac{a^{2}}{r_{+}^{2}} \sqrt{2(d-3)} .
$$

Notice how counterrotating orbits must be located very far away from the horizon as rotation increases. This is a consequence of having a "strong" ergoregion, extending through a large region in space.

For $d=5$ the previous equations simplify considerably, and we can find a simple solution:

$$
\begin{gathered}
r_{c}=\sqrt{2} \sqrt{\mu \pm a \sqrt{\mu}}, \\
\frac{1}{D_{c}}=\Omega_{c}=\frac{1}{-a \mp 2 \sqrt{\mu}} .
\end{gathered}
$$

\section{Circular timelike geodesics}

Timelike geodesics in a Myers-Perry spacetime are studied in Appendix B. In Sec. B 1 we show that for $d>$ 4 , there are no stable equatorial circular orbits in this spacetime. This extends the instability proof by Tangherlini [26] to rotating black holes, and the instability proof by Frolov and Stojkovic [48] to a general number of spacetime dimensions.

The energy and angular momentum of timelike circular geodesics are studied in Sec. B 2, where we also consider the orbital frequency $\Omega$ of general circular timelike geo- 
desics. In fact, it is possible to obtain a simple expression for the ratio $\Omega / \Omega_{c}$ [see Eq. (B25) in Appendix B]:

$$
\frac{\Omega}{\Omega_{c}}=\frac{\sqrt{2 r_{c}^{d-1}} \mp a \sqrt{(d-3) \mu}}{\sqrt{2 r^{d-1}} \mp a \sqrt{(d-3) \mu}} .
$$

This quantity is plotted in Fig. 1 for $\mu=2$ and selected values of $a$ and $d$. There is clearly a change in behavior for $d>5$ and large rotation, exemplified here for $d=6$. This will be explored in more detail in the next section.

\section{Lyapunov exponents}

Applying Eq. (19) to the case of Myers-Perry black holes we find

$$
\frac{\lambda}{\Omega_{c}}=\frac{\sqrt{d-1}\left(-\mu r_{c}^{4}+a^{2} r_{c}^{d-1}+r_{c}^{d+1}\right)}{a r_{c}^{d}+\sqrt{\frac{2 r_{c}^{d-1}}{\mu(d-3)}}\left(r_{c}^{d}-\mu r_{c}^{3}\right)} .
$$

Using Eq. (86) we can analytically compute the $d=5$ case, and we get

$$
\begin{gathered}
\lambda^{\text {counter }} \sqrt{\mu}=\sqrt{2} \frac{\sqrt{\mu-\sqrt{\mu} a}}{a-2 \sqrt{\mu}}, \\
\lambda^{\mathrm{co}} \sqrt{\mu}=\sqrt{2} \frac{\sqrt{\mu+\sqrt{\mu} a}}{a+2 \sqrt{\mu}} .
\end{gathered}
$$

The general case requires a numerical treatment. In Fig. 2 we show the Lyapunov exponent normalized by the angular velocity $\lambda / \Omega_{c}$ as a function of rotation $a$.

For corotating geodesics in $d=4$ and $d=5, \lambda$ asymptotes to zero near extremality $(a \rightarrow \mu / 2$ and $a \rightarrow \sqrt{\mu}$, respectively). This does not happen for $d>5$ and large rotation parameters: in this case, $\lambda / \Omega_{c}$ has a local minimum. This is consistent with QNM calculations in five [49] and higher dimensions [43]. The local minimum may be related to a change in behavior corresponding to a black hole $\rightarrow$ black brane transition, observed in [29] in

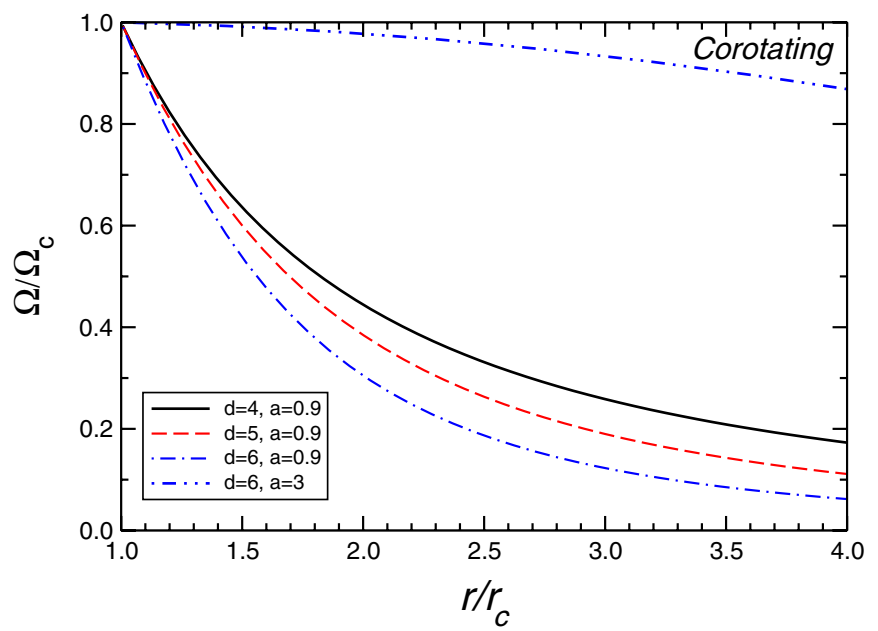

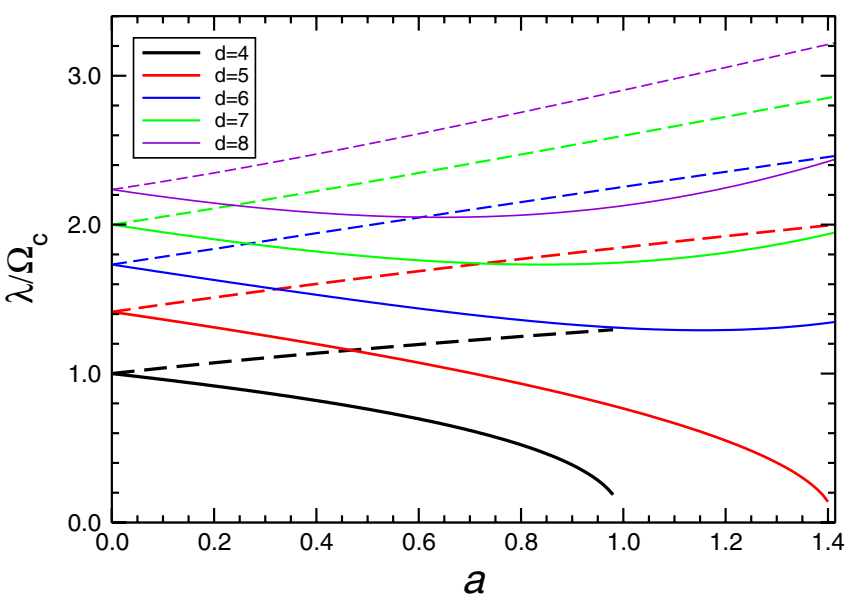

FIG. 2 (color online). Dimensionless instability exponents $\lambda / \Omega_{c}$ as a function of rotation for several spacetime dimensions $d$. We use units such that $\mu=2$. Solid lines refer to corotating orbits, dashed lines to counterrotating orbits.

relation with a conjectured instability of these systems for very large rotation rates.

The locations of minima in $\lambda / \Omega_{c}$ are given in Table I. In this table we also list a quantity considered in [29] as a possible indicator of a "transition" point, in this case the rotation at which the temperature has a minimum. The two

TABLE I. The transition point $a_{\text {trans }}$. The second column refers to the critical value of rotation $a$ for which the corotating Lyapunov exponent has a minimum. The third column refers to the point at which the temperature has a minimum, an indicator considered in [29].

\begin{tabular}{lcc}
\hline \hline$d$ & Lyapunov & Temperature \\
\hline 6 & 1.15 & 1.37 \\
7 & 0.84 & 1.28 \\
8 & 0.64 & 1.22 \\
50 & 0.05 & 1.02 \\
\hline \hline
\end{tabular}

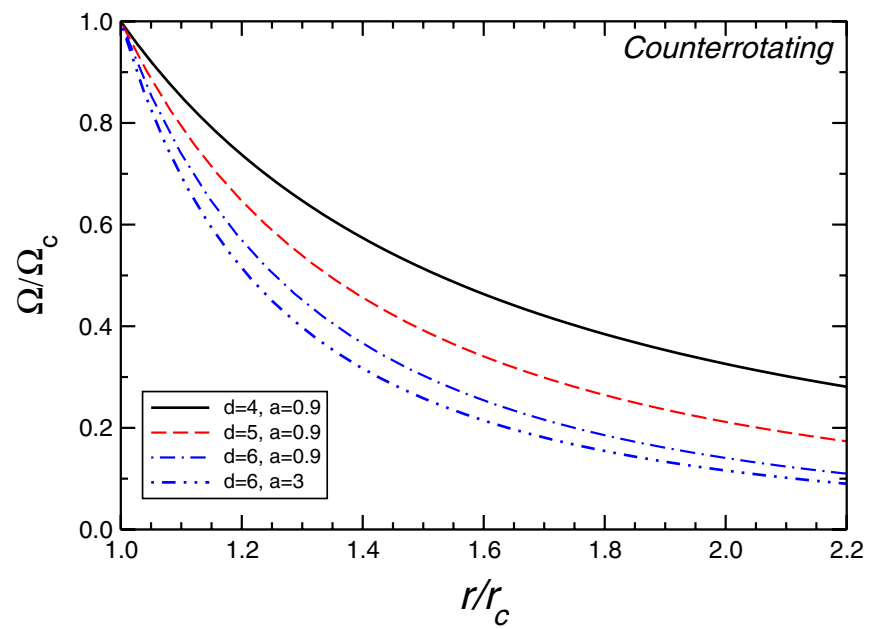

FIG. 1 (color online). Ratio of the timelike orbital frequency $\Omega$ to the orbital frequency $\Omega_{c}$ of corotating (left) and counterrotating (right) null geodesics as a function of $r / r_{c}$, with $r_{c}$ the radius of the null geodesic. 
quantities are roughly consistent for small $d$, but not for large $d$.

\section{CONCLUSIONS AND FUTURE WORK}

We have shown that for all spherically symmetric spacetimes, in a geometrical optics approximation, QNMs can be interpreted as particles trapped at unstable circular null geodesics and slowly leaking out. The leaking time scale is given by the principal Lyapunov exponent, for which we obtained a fairly simple expression, Eq. (19), in terms of the second derivative of the effective radial potential for geodesic motion. This simple, intuitive relation between QNMs and circular null geodesics is valid for all asymptotically flat, spherically symmetric black-hole spacetimes.

Some aspects of our investigation deserve further analysis. The interpretation of QNMs in terms of unstable circular null geodesics is valid in all generality only for spherically symmetric, asymptotically flat spacetimes. Once we break the azimuthal degeneracy, things get a bit more complex. For instance, in $d=4$ it is known that equatorial geodesics can account for the $l=|m|$ modes of Kerr-Newman black holes [10,12,50], but it is unclear whether this analogy can be extended to modes with $l \neq$ $|m|$. Perhaps modes with $l \neq m$ can be explained in terms of more general (e.g., nonequatorial) geodesics. Besides improving our intuitive physical understanding of ringdown radiation, a deeper exploration of this analogy could have important implications for the interpretation of numerical simulations of black-hole binary mergers and their use in gravitational-wave data analysis [51-53].

Another limitation of our results concerns their extension to non-asymptotically flat (e.g., anti-de Sitter) backgrounds. In the large damping limit, a certain class of QNMs has been associated with radial geodesics [5456]. It would be very interesting to extend this analysis to the eikonal (large- $l$ ) limit. A possible starting point could be the $2+1$-dimensional Bañados-Teitelboim-Zanelli black hole [57], for which QNM frequencies are known analytically [58]. Quite apart from the geodesic analogy, the large- $l$ limit is interesting per se. It turns out that the imaginary part of Schwsarzschild-anti-de Sitter QNMs decreases with $l$ [56]. Thus, if excited considerably, large- $l$ modes could dominate the black hole's response to perturbations. A more thorough investigation of the large- $l$ limit of QNMs is necessary.

Interesting physical phenomena could occur in (hypothetical) spacetimes for which timelike circular geodesics have a frequency equal to (or larger than) the frequency of unstable null geodesics. This would raise the interesting possibility of exciting QNMs by orbiting particles, possibly leading to instabilities of the spacetime. It would be interesting to find general conditions under which spacetimes possess stable null geodesics; stable null geodesics may also be associated with instability (or marginal stability) of spacetimes.

\section{ACKNOWLEDGMENTS}

We are grateful to Christopher Merrick and Frans Pretorius for sharing unpublished results with us. We thank Marc Casals, Curt Cutler, Paolo Pani and Jorge Rocha for a critical reading of the manuscript and useful suggestions. V.C.'s work was partially funded by Fundação para a Ciência e Tecnologia (FCT)-Portugal through Projects No. PTDC/FIS/64175/2006 and No. POCI/FP/81915/ 2007. A. S. M. thanks the CENTRA/IST for hospitality and financial help and the Conselho Nacional de Desenvolvimento Científico e Tecnológico (CNPq), Brazil, for a grant. H. W.'s work was partly supported by DFG Grant No. SFB/Transregio 7, Germany, and by Fundação para a Ciência e Tecnologia (FCT)-Portugal through Grant No. SFRH/BD/46061/2008 and Project No. PTDC/FIS/64175/2006. V. T.Z. is partially supported by Conselho Nacional de Desenvolvimento Científico e Tecnológico (CNPq)-Brazil. E. B.'s research was supported by the NASA Postdoctoral Program at JPL, administered by Oak Ridge Associated Universities through a contract with NASA.

\section{APPENDIX A: THE INSTABILITY TIME SCALE OF CIRCULAR GEODESICS: A SIMPLER DERIVATION}

Perhaps the simplest way to determine the instability time scale associated with circular null geodesics is through a consideration of the equations defining these geodesics $[5,10,11]$. Indeed, this was the approach originally adopted by Ferrari and Mashhoon to compute quasinormal modes of Kerr-Newman black holes in the eikonal limit $[10,11]$. In this appendix we will rederive the instability parameter $\lambda$ within their approach.

Consider small perturbations of a bundle of test null rays in the unstable equatorial circular orbit around a black hole described by the metric (22). First, rescale the affine parameter $s$ so as to be the coordinate time $t$, and consider the following values of the unperturbed geodesic:

$$
t=s, \quad r=r_{c}, \quad \theta=\frac{\pi}{2}, \quad \phi=\Omega_{c} s .
$$

The slightly perturbed equatorial null orbit is given by

$$
\begin{gathered}
s=t+\epsilon b(t), \\
r=r_{c}(1+\epsilon h(t)), \\
\theta=\pi / 2, \\
\phi=\Omega_{c}(t+\epsilon k(t)),
\end{gathered}
$$

where $|\epsilon| \ll 1$ denotes the dimensionless amplitude of the perturbation. Considering the leading terms in Eqs. (27)-(29), together with the boundary conditions that $b(t), h(t)$ and $k(t)$ vanish at $t=0$, yields 


$$
\begin{gathered}
h(t)=\sinh (\lambda t), \\
b(t)=\frac{r_{c} f_{c}^{\prime}}{E \lambda}(\cosh (\lambda t)-1), \\
k(t)=0 .
\end{gathered}
$$

The parameter $\lambda$ characterizes the decay rate and is determined by

$$
\lambda^{2}=\frac{f_{c}^{2}}{2 E^{2}} V_{r}^{\prime \prime}\left(r_{c}\right)=\frac{r_{c}^{2} f_{c}}{2 L^{2}} V_{r}^{\prime \prime}\left(r_{c}\right) .
$$

This result agrees with Eq. (40), obtained through the principal Lyapunov exponent.

Finally, the instability time scale can also be derived (at least in the usual Schwarzschild geometry) by considering a special class of geodesics: inspiralling geodesics that asymptote to the light ring when $t \rightarrow \infty$. These geodesics are considered in Chandrasekhar's book [34], and it is straightforward to compute how they approach the light ring.

\section{APPENDIX B: TIMELIKE GEODESICS IN THE EQUATORIAL PLANE OF MYERS-PERRY SPACETIMES}

In a $d$-dimensional Myers-Perry black hole with only one nonzero angular momentum parameter, the radial equation for geodesics in the equatorial plane can be cast in the form $r^{4} \dot{r}^{2}=V$, with $V \equiv r^{4} V_{r}$ as given in Eq. (72). The first derivative of $V$ with respect to $r$ is given by

$$
\begin{aligned}
V^{\prime}= & 4 r^{3} E^{2}-(d-5) \mu r^{4-d}(a E-L)^{2}+2 r\left(a^{2} E^{2}-L^{2}\right) \\
& -\delta_{1}\left[4 r^{3}+2 r a^{2}+(d-7) \mu r^{6-d}\right] .
\end{aligned}
$$

The conditions for the existence of circular orbits are $V=$ 0 and $V^{\prime}=0$ :

$$
\begin{aligned}
0 & =r^{2} E^{2}+\mu r^{3-d}(a E-L)^{2}+\left(a^{2} E^{2}-L^{2}\right)-\delta_{1} \Delta, \\
0= & 4 r^{2} E^{2}-(d-5) \mu r^{3-d}(a E-L)^{2}+2\left(a^{2} E^{2}-L^{2}\right) \\
& -\delta_{1}\left(2 \Delta+r \Delta^{\prime}\right) .
\end{aligned}
$$

Eliminating the term $a^{2} E^{2}-L^{2}$ one finds

$$
2 r E^{2}-(d-3) \mu r^{2-d}(a E-L)^{2}-\Delta^{\prime} \delta_{1}=0 .
$$

\section{The instability of geodesics in the equatorial plane of Myers-Perry spacetimes}

Equations (B2) and (B4) will be used here to obtain the values of energy $E$ and angular momentum $L$ associated to circular timelike $\left(\delta_{1}=1\right)$ geodesics. Using (B4) and introducing the new quantities $x=L-a E$ and $M=\mu / 2$, and the reciprocal radius $u=1 / r$, we obtain an expression for $E^{2}$ :

$$
E^{2}=\left[1-(5-d) M u^{d-3}\right]+(d-3) M u^{d-1} x^{2} .
$$

With this expression for $E^{2}$, Eq. (B2) leads to

$$
\begin{aligned}
2 a E x u= & x^{2}\left[(d-1) M u^{d-3}-1\right] u \\
& -\left[a^{2} u-(d-3) M u^{d-4}\right] .
\end{aligned}
$$

For $d=4$ spacetime dimensions, the above equations are identical to those obtained by Chandrasekhar [34]. We can now eliminate $E$ in Eqs. (B5) and (B6) to obtain a quadratic equation for $x^{2}$ :

$$
\begin{aligned}
0= & x^{4} u^{2}\left[\left[(d-1) M u^{d-3}-1\right]^{2}-4 a^{2} M(d-3) u^{d-1}\right] \\
& -2 x^{2} u\left[\left[(d-1) M u^{d-3}-1\right]\left[a^{2} u-(d-3) M u^{d-4}\right]\right. \\
& \left.-2 a^{2} u\left[(5-d) M u^{d-3}-1\right]\right]+\left[a^{2} u-(d-3) M u^{d-4}\right]^{2} .
\end{aligned}
$$

The discriminant associated to this equation is given by $16 M a^{2}(d-3) u^{d-1} \Delta_{u}^{2}$, where we have introduced $\Delta_{u}=$ $a^{2} u^{2}-2 M u^{d-3}+1$. In order to write the solutions of Eq. (B7), it is convenient to consider the expression

$$
\left[(d-1) M u^{d-3}-1\right]^{2}-4 a^{2} M(d-3) u^{d-1}=Q_{-} Q_{+},
$$

where

$$
Q_{ \pm}=1-(d-1) M u^{d-3} \pm 2 a \sqrt{(d-3) M u^{d-1}} .
$$

Then the solutions of Eq. (B7) can be written as

$$
x^{2} u^{2}=\frac{Q_{ \pm} \Delta_{u}-Q_{+} Q_{-}}{Q_{+} Q_{-}}=\frac{1}{Q_{\mp}}\left(\Delta_{u}-Q_{\mp}\right) .
$$

As an alternative, we can use the identity

$$
\Delta_{u}-Q_{\mp}=u\left[a \sqrt{u} \pm \sqrt{(d-3) M u^{d-4}}\right]^{2}
$$

to cast the solution for $x$ in the simple form

$$
x=-\frac{a \sqrt{u} \pm \sqrt{(d-3) M u^{d-4}}}{\sqrt{u Q_{\bar{\mp}}}},
$$

where the upper sign in the foregoing equations applies to counterrotating orbits, while the lower sign applies to corotating orbits. Inserting expression (B12) in Eq. (B5) and using the relation $L=a E+x$, we get the following expression for $E$ :

$$
E=\frac{1}{\sqrt{Q_{\mp}}}\left[1-2 M u^{d-3} \mp a \sqrt{(d-3) M u^{d-1}}\right],
$$

and the angular momentum associated with the circular geodesics,

$$
L=\mp \frac{\sqrt{(d-3) M u^{d-4}}}{\sqrt{u Q_{\mp}}}\left[1+a^{2} u^{2} \pm 2 a \sqrt{\frac{M u^{d-1}}{d-3}}\right] .
$$


In order to investigate the stability of circular timelike orbits we must compute the second derivative of $V$ with respect to $r$ for the values of $E$ and $L$ specific to circular orbits. Differentiating Eq. (B1) we find

$$
\begin{aligned}
V^{\prime \prime}= & 12 r^{2}\left(E^{2}-1\right)+2(d-4)(d-5) M r^{3-d} x^{2}-2 x^{2} \\
& -4 a E x-2 a^{2}+2(d-6)(d-7) M r^{5-d} .
\end{aligned}
$$

Substituting $x$ and $E$ from Eqs. (B12) and (B13), the above expression for $V^{\prime \prime}$ becomes

$$
\begin{aligned}
V^{\prime \prime}= & \frac{2(d-3) M u^{d-5}}{Q_{\mp}}\left[2(d-1) M u^{d-3}+(d-5)\right. \\
& \left. \pm 8 a \sqrt{(d-3) M u^{d-1}}+(d-1) a^{2} u^{2}\right] .
\end{aligned}
$$

The term within square brackets in the foregoing equation is equal to $(d-1) \Delta_{u}-4 Q_{\mp}$, so that the second derivative of $V$ reduces to

$$
V^{\prime \prime}=\frac{2(d-3) M u^{d-5}}{Q_{\mp}}\left[(d-1) \Delta_{u}-4 Q_{\mp}\right] .
$$

This expression shows an explicit dependence on the spacetime dimensionality $d$. To analyze the sign of $V^{\prime \prime}$ in Eq. (B17) it will be helpful to distinguish between different values of $d$. Since $E, L$ and $x=L-a E$ must be real, the functions $\Delta_{u}$ and $Q_{ \pm}$are such that

$$
\Delta_{u} \geq Q_{ \pm} \geq 0 .
$$

For $d \geq 5$ the above conditions lead to

$$
(d-1) \Delta_{u} \geq 4 Q_{\mp} \Rightarrow V^{\prime \prime} \geq 0 .
$$

This means that there are no stable timelike circular orbits for spacetimes with $d \geq 5$. This generalizes previous work by Tangherlini on nonrotating higher-dimensional black holes [26] and by Frolov and Stojkovic on five-dimensional rotating black holes [48].

\section{The orbital frequency of circular geodesics}

The orbital frequency $\Omega=d \varphi / d t$ associated to circular timelike geodesics is given by

$$
\Omega=\frac{\left(L-2 M u^{d-3} x\right) u^{2}}{\left(1+a^{2} u^{2}\right) E-2 a M u^{d-1} x} .
$$

The foregoing expression can be simplified by considering the following identities:

$$
\begin{gathered}
L-2 M u^{d-3} x=\mp \frac{\sqrt{(d-3) M u^{d-4}}}{\sqrt{u Q_{\mp}}} \Delta_{w}, \\
\left(1+a^{2} u^{2}\right) E-2 a M u^{d-1} x \\
=\frac{\Delta_{u}}{\sqrt{Q_{\mp}}}\left[1 \mp a \sqrt{(d-3) M u^{d-1}}\right] .
\end{gathered}
$$

Substituting (B21) and (B22) into Eq. (B20), we obtain

$$
\Omega=\frac{\mp \sqrt{(d-3) M u^{d-1}}}{1 \mp a \sqrt{(d-3) M u^{d-1}}} .
$$

By considering Eq. (79) for $D_{c}$ and the relation $\Omega_{c}=$ $1 / D_{c}$, we find a similar expression for circular null geodesics:

$$
\Omega_{c}=\frac{\mp \sqrt{(d-3) M u_{c}^{d-1}}}{1 \mp a \sqrt{(d-3) M u_{c}^{d-1}}} .
$$

Consequently, the ratio $\Omega / \Omega_{c}$ varies with $r$ as follows:

$$
\frac{\Omega}{\Omega_{c}}=\frac{\sqrt{r_{c}^{d-1}} \mp a \sqrt{(d-3) M}}{\sqrt{r^{d-1}} \mp a \sqrt{(d-3) M}} .
$$

\section{APPENDIX C: SCHWARZSCHILD-ANTI- DE SITTER SPACETIMES}

In this appendix we consider Schwarzschild-antide Sitter spacetimes, and we show that the analogy between unstable circular orbits and black-hole QNMs is not trivially extended to non-asymptotically flat backgrounds. A higher-dimensional Schwarzschild-anti-de Sitter solution is a solution of

$$
G_{a b}+\frac{(d-2)(d-1)}{2 L_{\mathrm{ads}}} g_{a b}=0,
$$

with cosmological constant $\Lambda \equiv-(d-2)(d-1) /\left(2 L_{\text {ads }}\right)$ and typical curvature radius $L_{\text {ads }}$. We consider the simplest black-hole solution: the $d$-dimensional Schwarzschildanti-de Sitter solution. The line element is given by Eq. (22) with

$$
f(r)=g(r)=\left(\frac{r^{2}}{L_{\mathrm{ads}}^{2}}+1-\frac{r_{0}^{d-3}}{r^{d-3}}\right) .
$$

The quantity $r_{0}$ is related to the mass $M$ of the spacetime,

$$
M=\frac{(d-2) A_{d-2} r_{0}^{d-3}}{16 \pi},
$$

and the horizon radius $r_{+}$is the largest real root of $f(r)=$ 0 .

For circular null geodesics, an exact solution can be found with

$$
r_{c}=2^{1 /(3-d)}\left[(d-1)\left(1+\frac{r_{+}^{2}}{L_{\text {ads }}^{2}}\right)\right]^{1 /(3-d)} r_{+} .
$$

Since $V_{r}^{\prime \prime}\left(r_{c}\right)=L^{2}(2 d-6) r_{c}^{-4}$, circular null geodesics are unstable. The angular velocity at this radius is given by

$$
\begin{aligned}
L_{\mathrm{ads}}^{2} \Omega_{c}^{2}= & +\frac{(d-3)}{2}\left(\frac{2}{d-1}\right)^{(d-1) /(d-3)} \\
& \times\left(1+\frac{r_{+}^{2}}{L_{\mathrm{ads}}^{2}}\right)^{-2 /(d-3)} \frac{L_{\mathrm{ads}}^{2}}{r_{+}^{2}}
\end{aligned}
$$

and reduces to $\Omega_{c} \approx 1 / L_{\text {ads }}$ for large $\left(r_{+} / L_{\text {ads }} \gg 1\right)$ black 
holes. The calculation of the instability exponents proceeds trivially. As in the case of higher-dimensional Schwarzschild black holes, Eq. (50), we find

$$
\lambda / \Omega_{c}=\sqrt{d-3} .
$$

If our main result, Eq. (46), were valid in asymptotically anti-de Sitter (AdS) spacetimes, in the eikonal limit we would get $\omega_{\mathrm{QNM}} / \Omega_{c}=l-i \sqrt{d-3}(n+1 / 2)$. However, according to both analytical [56] and numerical results [59-62], the imaginary part of QNM frequencies in this background increases monotonically with increasing $r_{+}$. This dependence on $r_{+}$cannot be explained by the circular null geodesic analogy. In hindsight, this failure is not too surprising. AdS spacetimes are not globally hyperbolic; boundary conditions at infinity must be taken into account. On the other hand, geodesic calculations are local, and they carry no information about spatial infinity. The available analytical and numerical results [56,59-62] indicate that the damping time scale is smaller than indicated by the geodesic calculation, Eq. (62). Perhaps the disagreement could be explained by arguing that null particles reach spatial infinity on time scales faster than the geodesic time scale, and therefore one would have to correct for this.
[1] R. Narayan, New J. Phys. 7, 199 (2005).

[2] S. N. Zhang, W. Cui, and W. Chen, Astrophys. J. 482, L155 (1997).

[3] S. L. Shapiro and S. A. Teukolsky, Black Holes, White Dwarfs, and Neutron Stars: The Physics of Compact Objects (Wiley, New York, 1983).

[4] M. A. Podurets, Astron. Zh. 41, 1090 (1964) [Sov. Astron. 8, 868 (1965)].

[5] W. L. Ames and K.S. Thorne, Astrophys. J. 151, 659 (1968).

[6] Kostas D. Kokkotas and Bernd Schmidt, Living Rev. Relativity 2, 2 (1999), http://www.livingreviews.org/lrr1999-2.

[7] H. P. Nollert, Classical Quantum Gravity 16, R159 (1999).

[8] W. H. Press, Astrophys. J. 170, L105 (1971).

[9] C. J. Goebel, Astrophys. J. 172, L95 (1972).

[10] V. Ferrari and B. Mashhoon, Phys. Rev. D 30, 295 (1984).

[11] B. Mashhoon, Phys. Rev. D 31, 290 (1985).

[12] E. Berti and K. D. Kokkotas, Phys. Rev. D 71, 124008 (2005).

[13] L. Bombelli and E. Calzetta, Classical Quantum Gravity 9 , 2573 (1992).

[14] N. J. Cornish and J. J. Levin, Classical Quantum Gravity 20, 1649 (2003).

[15] F. Pretorius and D. Khurana, Classical Quantum Gravity 24, S83 (2007).

[16] G. Perez-Giz and J. Levin, arXiv:0811.3815.

[17] J. Levin and G. Perez-Giz, arXiv:0811.3814.

[18] R. Grossman and J. Levin, arXiv:0811.3798 [Phys. Rev. D (to be published)].

[19] A. F. Steklain and P. S. Letelier, Phys. Lett. A 373, 188 (2009).

[20] U. Sperhake, V. Cardoso, F. Pretorius, E. Berti, and J. A. Gonzalez, Phys. Rev. Lett. 101, 161101 (2008).

[21] M. Shibata, H. Okawa, and T. Yamamoto, Phys. Rev. D 78, 101501 (2008).

[22] B. Mashhoon, in Proceedings of the Third Marcel Grossmann Meeting on Recent Developments of General Relativity, Shanghai, 1982, edited by $\mathrm{Hu}$ Ning (NorthHolland, Amsterdam, 1983).

[23] B.F. Schutz and C. M. Will, Astrophys. J. 291, L33
(1985).

[24] S. Iyer and C. M. Will, Phys. Rev. D 35, 3621 (1987).

[25] S. Iyer, Phys. Rev. D 35, 3632 (1987).

[26] F. R. Tangherlini, Nuovo Cimento 27, 636 (1963).

[27] V. Cardoso and J.P. S. Lemos, Phys. Rev. D 67, 084020 (2003).

[28] R. C. Myers and M. J. Perry, Ann. Phys. (N.Y.) 172, 304 (1986).

[29] R. Emparan and R. C. Myers, J. High Energy Phys. 09 (2003) 025.

[30] J. M. Bardeen, W.H. Press, and S.A. Teukolsky, Astrophys. J. 178, 347 (1972).

[31] J.R. Dorfman, An Introduction to Chaos in Nonequilibrium Statistical Mechanics (Cambridge University Press, Cambridge, England, 1999); H. A. Posh and W. G. Hoover, J. Phys. Conf. Ser. 31, 9 (2006).

[32] C. Skokos, arXiv:0811.0882.

[33] J. M. Stewart, Proc. R. Soc. A 424, 239 (1989).

[34] S. Chandrasekhar, The Mathematical Theory of Black Holes (Oxford University Press, New York, 1983).

[35] H. Kodama and A. Ishibashi, Prog. Theor. Phys. 110, 701 (2003).

[36] A. Ishibashi and H. Kodama, Prog. Theor. Phys. 110, 901 (2003).

[37] H. Kodama and A. Ishibashi, Prog. Theor. Phys. 111, 29 (2004).

[38] V. M. Rosa and P. S. Letelier, Phys. Rev. D 78, 084038 (2008).

[39] C. Merrick and F. Pretorius (unpublished).

[40] A.S. Barreto and M. Zworski, Math. Res. Lett. 4, 103 (1997).

[41] R. A. Konoplya, Phys. Rev. D 68, 024018 (2003).

[42] E. Berti, M. Cavaglia, and L. Gualtieri, Phys. Rev. D 69, 124011 (2004).

[43] V. Cardoso, G. Siopsis, and S. Yoshida, Phys. Rev. D 71, 024019 (2005).

[44] D. Ida, K. y. Oda, and S. C. Park, Phys. Rev. D 67, 064025 (2003); 69, 049901(E) (2004).

[45] D. Ida, K. y. Oda, and S. C. Park, Phys. Rev. D 73, 124022 (2006).

[46] D. Ida, K. y. Oda, and S. C. Park, Phys. Rev. D 71, 124039 

(2005).

[47] V. Cardoso and S. Yoshida, J. High Energy Phys. 07 (2005) 009.

[48] V.P. Frolov and D. Stojkovic, Phys. Rev. D 68, 064011 (2003).

[49] E. Berti, K. D. Kokkotas, and E. Papantonopoulos, Phys. Rev. D 68, 064020 (2003).

[50] E. Berti, V. Cardoso, and C. M. Will, Phys. Rev. D 73, 064030 (2006).

[51] E. Berti, V. Cardoso, J. A. Gonzalez, U. Sperhake, M. Hannam, S. Husa, and B. Bruegmann, Phys. Rev. D 76, 064034 (2007).

[52] C. Hanna, M. Megevand, E. Ochsner, and C. Palenzuela, arXiv:0801.4297.

[53] J. G. Baker, W. D. Boggs, J. Centrella, B. J. Kelly, S. T. McWilliams, and J. R. van Meter, Phys. Rev. D 78, 044046 (2008).
[54] L. Fidkowski, V. Hubeny, M. Kleban, and S. Shenker, J. High Energy Phys. 02 (2004) 014.

[55] I. Amado and C. Hoyos-Badajoz, J. High Energy Phys. 09 (2008) 118.

[56] G. Festuccia and H. Liu, arXiv:0811.1033.

[57] M. Bañados, C. Teitelboim, and J. Zanelli, Phys. Rev. Lett. 69, 1849 (1992).

[58] V. Cardoso and J. P. S. Lemos, Phys. Rev. D 63, 124015 (2001).

[59] G. T. Horowitz and V. E. Hubeny, Phys. Rev. D 62, 024027 (2000).

[60] V. Cardoso, R. Konoplya, and J. P. S. Lemos, Phys. Rev. D 68, 044024 (2003).

[61] A. S. Miranda and V. T. Zanchin, Phys. Rev. D 73, 064034 (2006).

[62] E. Berti and K. D. Kokkotas, Phys. Rev. D 68, 044027 (2003). 